\title{
PROPERTIES OF VECTOR VALUED FINITELY ADDITIVE SET FUNCTIONS
}

\author{
R. B. DARST ${ }^{1}$
}

Suppose that $\Sigma$ is an algebra of subsets of a set $S$. If $B$ is a Banach space over the real numbers $R$, then $H(B)=H(S, \Sigma, B)$ denotes the space of bounded $\left(\sup _{E \in \Sigma}\|\beta(E)\|_{B}<\infty\right), B$-valued, finitely additive set functions $\beta$ on $\Sigma$. Suppose that $B$ has a basis $\left\{b_{i}\right\}$ of unit vectors. Then the coefficient functionals $\beta_{i}, i \geqq 1$, of $\beta$, determined by $\beta(E)$ $=\sum b_{i} \beta_{i}(E)$, are elements of $H(R)$. The purpose of this paper is to initiate a study of the interplay between $\beta$, the sequence $\left\{\beta_{i}\right\}$, and the basis $\left\{b_{i}\right\}$. Properties which are obtained are used to establish some Radon-Nikodým theorems relating $\beta \in H(B)$ and $\alpha \in H(A), A$ a Banach space over $R$.

Let us begin by recalling that because $\left\{b_{i}\right\}$ is a basis for $B$ and $\left\|b_{i}\right\|=1, i \geqq 1$, it follows [8, p. 67, Theorem 1] that there exists $M_{B}>0$ such that if $\sum b_{i} \lambda_{i} \in B$, then $\left\|\sum_{i \unlhd n} b_{i} \lambda_{i}\right\| \leqq M_{B}\left\|\sum b_{i} \lambda_{i}\right\|$ and, hence, $\left\|\sum_{m<i \leqq n} b_{i} \lambda_{i}\right\| \leqq 2 M_{B}\left\|\sum b_{i} \lambda_{i}\right\|$. In particular, $\left|\lambda_{i}\right| \leqq 2 M_{B}\left\|\sum b_{i} \lambda_{i}\right\|$, $i \geqq 1$. Thus the coefficient functionals $\beta_{i}$ of $\beta$ are bounded by $2 M_{B}|\beta|{ }_{s}(S)$ where $|\beta|_{8}(S)=\sup \{\|\beta(E)\| ; E \in \Sigma, E \subset S\}$. Since the functionals $\beta_{i}$ are also finitely additive, $\left\{\beta_{i}\right\}$ is a bounded sequence of elements of $H(R)$. Moreover, since $\sum b_{i} \lambda_{i} \in B$ implies that $\lim \lambda_{i}=0$, it follows that $\lim \beta_{i}(E)=0, E \in \Sigma$. This latter property permits us to assert that the sequence $\left\{\beta_{i}\right\}$ converges weakly to zero if $\Sigma$ is a sigma algebra [6]. The following example shows that, in general, this is not the case.

Example 1. Let $e_{i}=\left\{\delta_{i j}\right\}_{j \geqq 1}, \delta_{i j}=1$ if $i=j$ and $\delta_{i j}=0$ if $i \neq j$. Then $\left\{e_{i}\right\}$ is a sequence of unit vectors which is a basis for the space $c_{0}$ of sequences of real numbers which converge to zero. Let $\Sigma_{0}$ be an algebra of subsets of a set $S_{0}$ such that there exists a bounded sequence $\left\{\gamma_{i}\right\}$ of elements of $H\left(S_{0}, \Sigma_{0}, R\right)$ satisfying

(1) $\lim _{i} \gamma_{i}(E)=0, E \in \Sigma_{0}$ and

(2) the sequence $\left\{\gamma_{i}\right\}$ does not converge weakly to zero.

Let $\gamma \in H\left(S_{0}, \Sigma_{0}, c_{0}\right)$ be defined by $\gamma(E)=\sum e_{i} \gamma_{i}(E)$.

Example 1 shows that if we wish to conclude that $\left\{\beta_{i}\right\}$ converges weakly to zero, then we must impose additional conditions somewhere. We shall first consider a condition on $\beta$ and then conditions on $B$. The condition which we shall impose on $\beta$ is a natural condition

Received by the editors May 20, 1968.

1 The author was partially supported by a NSF grant. 
to impose when seeking to compare $\beta$ with an element $\alpha$ of $H(A)$ (see [4, particularly the concluding remarks]). The conditions, $G_{1}$ and $G_{2}$, on $B$ are technical conveniences which, fortunately, are of sufficiently common occurrence to warrant their introduction.

Before proceeding to Theorem 1 , let us recall that if $\mu \in H(R)$ and $E \in \Sigma$, then $v(\mu, E)=\sup \{\mu(F) ; F \in \Sigma, F \subset E\}-\inf \{\mu(F) ; F \in \Sigma, F \subset E\}$ is the variation of $\mu$ on $E$.

Theorem 1. Suppose that $\lim \left\|\beta\left(F_{j}\right)\right\|=0$ whenever $\left\{F_{j}\right\}$ is a sequence of pairwise disjoint elements of $\Sigma$. Then the sequence $\left\{\beta_{i}\right\}$ converges weakly to zero.

Proof. Suppose, on the contrary, that $\left\{\beta_{i}\right\}$ does not converge weakly to zero. Then, by Theorem 3.1 of [11] (cf. [5]), there exists $\epsilon>0$ and a sequence $\left\{E_{p}\right\}$ of pairwise disjoint elements of $\Sigma$ such that $\left|\sum_{p} \beta_{i}\left(E_{p}\right)\right|>3 \epsilon$ for infinitely many positive integers $i$. Let $i_{1}$ be the least such positive integer, and let $p_{1}$ be the least positive integer satisfying $\left|\sum_{p \leqq p_{1}} \beta_{i_{1}}\left(E_{p}\right)\right|>3 \epsilon$. Let $F_{1}=\bigcup_{p_{\S} p_{1}} E_{p}$. The following facet of this construction is not necessary to establish Theorem 1 but will be convenient for our proof of Theorem 2. Let $q_{1}$ be the least positive integer greater than $p_{1}$ for which $\sum_{p \geq q_{1}} v\left(\beta_{i_{1}}, E_{p}\right)<\epsilon$. Let $k_{1}$ be the least positive integer such that $i \geqq k_{1}$ implies that $\sum_{p<q_{1}}\left|\beta_{i}\left(E_{p}\right)\right|<\epsilon$. This completes the first stage of this construction; to set the pattern, the second stage follows. Let $i_{2}$ be the least positive integer greater than $k_{1}\left(k_{1} \geqq i_{1}\right)$ such that $\left|\sum_{p} \beta_{i_{2}}\left(E_{p}\right)\right|>3 \epsilon$, and let $p_{2}$ be the least positive integer satisfying $\left|\sum_{p \leqq p_{2}} \beta_{i_{2}}\left(E_{p}\right)\right|>3 \epsilon$. Let $F_{2}=\bigcup_{q_{1} \leqq p \leqq p_{2}} E_{p}$. Then $\left|\beta_{i_{2}}\left(F_{2}\right)\right|>\left|\sum_{p \leqq p_{2}} \beta_{i_{2}}\left(E_{p}\right)\right|-\sum_{p<q_{1}}\left|\beta_{i_{2}}\left(E_{p}\right)\right|>2 \epsilon$. Let $q_{2}$ be the least positive integer greater than $p_{2}$ such that $\sum_{p \geq q_{2}} v\left(\beta_{i_{2}}, E_{p}\right)<\epsilon$. Let $k_{2}$ be the least positive integer such that $i>k_{2}$ implies that $\sum_{p<q_{2}}\left|\beta_{i}\left(E_{p}\right)\right|<\epsilon$. Repeating this process inductively, we obtain a sequence $\left\{F_{i}\right\}$ of pairwise disjoint elements of $\Sigma$ and an increasing sequence $i_{1}, i_{2}, \ldots$ of positive integers such that

(1) $\beta_{i_{j}}\left(F_{j}\right)>2 \epsilon$ and

(2) if $k \geqq j$, then

$$
\begin{aligned}
\left|\beta_{i_{j}}\left(\bigcup_{n \leqq k} F_{n}\right)\right| \geqq & -\sum_{p<q_{k-1}}\left|\beta_{i_{j}}\left(E_{p}\right)\right| \\
& +\left|\sum_{q_{j-1} \leqq p \leqq p_{j}} \beta_{i_{j}}\left(E_{p}\right)\right|-\sum_{p \geqq q_{j}} v\left(\beta_{i_{j}}, E_{p}\right)>\epsilon .
\end{aligned}
$$

Notice that up to now we have not used the hypothesis of Theorem 1. Invoking now the hypothesis $\left\|\beta\left(F_{j}\right)\right\| \rightarrow 0$, a contradiction obtains from (1) and $\left|\beta_{i_{j}}\left(F_{j}\right)\right| \leqq 2 M_{B}\left\|\beta\left(F_{j}\right)\right\|$. 
The following example shows that the converse to Theorem 1 is not true.

ExAmple 2. Suppose that $N$ is the set of positive integers and that $\Gamma$ is the sigma algebra of all subsets of $N$. Suppose that $L$ is the linear span, over $R$, of the sequences $e_{i}=\left\{\delta_{i j}\right\}_{j \geqq 1}$, is normed by

$\left\|\{x\}_{i}\right\|=\sup \left\{\left|x_{1}\right|,\left(\left|x_{2}\right|+\left|x_{3}\right|\right),\left(\left|x_{4}\right|+\left|x_{5}\right|+\left|x_{6}\right|\right), \cdots\right\}$,

and let $X$ denote the completion of $L$. Then $X$ is represented as a space of sequences. Moreover, because $\lim x_{i}=0$, whenever $x=\left\{x_{i}\right\}$ $\in X$, it follows that $\left\{e_{i}\right\}$ is a basis for $X$. For $i \geqq 1$, let $\beta_{i} \in H(N, \Gamma, R)$ be defined by $\beta_{i}(E)=1 / k$ if $i \in E_{k}=\{i:(k-1) k<2 i \leqq k(k+1)\}$ and $i \in E$, and $\beta_{i}(E)=0$ otherwise. Let $\beta(E)=\sum e_{i} \beta_{i}(E)$. Then $\beta \in H(N, \Gamma, X)$ and, hence because $\Gamma$ is a sigma algebra, $\left\{\beta_{i}\right\}$ converges weakly to zero. Moreover, $\lim v\left(\beta_{i}, S\right)=0$ which of course, also implies weak convergence to zero. Nevertheless, $\left\{E_{k}\right\}$ is a sequence of pairwise disjoint elements of $\Gamma$ and $\left\|\beta\left(E_{k}\right)\right\|=1, k \geqq 1$.

If $\left\{\beta_{i}\right\}$ is a sequence of elements of $H(R)$ satisfying $\Sigma v\left(\beta_{i}, S\right)<\infty$ and $\beta$ is defined by $\beta(E)=\sum b_{i} \beta_{i}(E)$, then $\beta \in H(B)$ and $\lim \left\|\beta\left(E_{i}\right)\right\|=0$ whenever $\left\{E_{i}\right\}$ is a sequence of pairwise disjoint elements of $\Sigma$. (Even more is true, namely, $\Sigma\left\|\beta\left(E_{i}\right)\right\|<\infty$.) Thus additional conditions on $B$ are not necessary in order that the hypothesis of Theorem 1 be satisfied. We shall introduce two growth conditions on a Banach space with basis and show (Theorem 3 ) that one of them implies the hypothesis of Theorem 1.

The statement that $\left\{b_{i}\right\}$ satisfies condition $G_{1}$ means that if each of $M$ and $\epsilon$ is a positive number, then there is a positive number $\delta=\delta(M, \epsilon)$ such that if $n$ is a positive integer, $x=\sum_{i \leqq n} b_{i} \lambda_{i}$, $y=b_{n+1} \lambda_{n+1},\|x\| \leqq M$, and $\|y\| \geqq \epsilon$, then $\|x+y\| \geqq\|x\|+\delta$.

Notice that if condition $G_{1}$ is satisfied, then $\left\{b_{i}\right\}$ is a monotone basis: $\left\|\sum_{i \leqq n} b_{i} \lambda_{i}\right\| \leqq\left\|\sum_{i \leqq n+1} b_{i} \lambda_{i}\right\|$. We might also mention that $\delta(M, \epsilon)=M \delta(1, \epsilon / M)$.

The statement that $\left\{b_{i}\right\}$ satisfies condition $G_{2}$ means that if $(M, \epsilon)$ is a pair of positive numbers, then there is a positive number $\gamma(M, \epsilon)$ such that if $1 \leqq n<p, x=\sum_{i \leqq n} b_{i} \lambda_{i}, y=\sum_{n<i \leqq p} b_{i} \lambda_{i},\|x\| \leqq M$, and $\|y\| \geqq \epsilon$, then $\|x+y\| \geqq\|x\|+\gamma(M, \epsilon)$.

It is clear that if $\left\{b_{i}\right\}$ satisfies $G_{2}$, then it also satisfies $G_{1}(\delta(M, \epsilon)$ $\geqq \gamma(M, \epsilon))$. We are unaware of an example where $G_{1}$ is satisfied but $G_{2}$ is not. We shall next show that if $G_{1}$ is satisfied, then $\left\{\beta_{i}\right\}$ converges weakly to zero. Then we shall show that if $G_{2}$ is satisfied, then the hypothesis of Theorem 1 is satisfied.

Theorem 2. Suppose that $\left\{b_{i}\right\}$ satisfies condition $G_{1}$. Then the coefficient functionals $\beta_{i}$ converge weakly to zero. 
Proof. Suppose again that $\left\{\beta_{i}\right\}$ does not converge weakly to zero. Then all but the last sentence of the proof of Theorem 1 applies. Let $M=M_{B}|\beta|_{s}(S)$. Let $k$ be a positive integer satisfying $k \delta>M$, and let $E=\bigcup_{j \leqq k} F_{j}$. Then the contradiction $M<\delta k<\sum_{j \leqq k}\left(\left\|\sum_{n \leqq i} b_{n} \beta_{n}(E)\right\|\right.$ $\left.-\left\|\sum_{n<i_{j}} b_{n} \beta_{n}(E)\right\|\right) \leqq\left\|\sum_{n \leqq i_{k}} b_{n} \beta_{n}(E)\right\| \leqq M_{B}\|\beta(E)\| \leqq M_{B}|\beta|{ }_{s}(S)$ follows from (2) of the proof of Theorem 1 together with the hypothesis of Theorem 2.

Theorem 3. Suppose that $\left\{b_{i}\right\}$ satisfies condition $G_{2}$. Then $\lim _{i}\left\|\beta\left(E_{i}\right)\right\|=0$ whenever $\left\{E_{i}\right\}$ is a sequence of pairwise disjoint elements of $\Sigma$.

Proof. Suppose on the contrary that there exists $\epsilon>0$ and a sequence $\left\{E_{n}\right\}$ of pairwise disjoint element of $\Sigma$ such that $\left\|\beta\left(E_{n}\right)\right\|>3 \epsilon$, $n \geqq 1$. Then there exists a positive integer $k_{1}$ such that $\left\|\sum_{i \leq k_{1}} b_{i} \beta_{i}\left(E_{1}\right)\right\|$ $>3 \epsilon$ and, moreover, if $k_{1} \leqq p<n$, then $\left\|\sum_{p<i \leqq n} b_{i} \beta_{i}\left(E_{1}\right)\right\|<\epsilon / 2$. Let $n_{1}=1$. There exists a positive integer $n_{2}$ such that $\sum_{i \leqq k_{1}} \sum_{j \geqq n_{2}}$ $v\left(\beta_{i}, E_{j}\right)<\epsilon$. There exists a positive integer $k_{2}$ such that

$$
\left\|\sum_{i \leqq k_{2}} b_{i} \beta_{i}\left(E_{n_{2}}\right)\right\|>3 \epsilon
$$

and, moreover, if $k_{2} \leqq p<n$, then $\left\|\sum_{p<i \leqq n} b_{i} \beta_{i}\left(E_{n_{2}}\right)\right\|<\epsilon / 2^{2}$. There exists a positive integer $n_{3}$ such that $\sum_{i \leqq k_{2}} \sum_{j \geqq n_{3}} v\left(\beta_{i}, E_{j}\right)<\epsilon$. Iterate this procedure. Let $m$ be a positive integer. Then

$$
\left\|\sum_{i \leqq k_{1}} b_{i} \beta_{i}\left(\bigcup_{j \leqq m} E_{n_{j}}\right)\right\| \geqq\left\|\sum_{i \leqq k_{1}} b_{i} \beta_{i}\left(E_{n_{1}}\right)\right\|-\sum_{i \leqq k_{1}} \sum_{j \geqq n_{2}} v\left(\beta_{i}, E_{j}\right)>2 \epsilon .
$$

For $p>1$, let $S_{p}=\left\{i ; k_{p-1}<i \leqq k_{p}\right\}$. Then, for $1<p<m$,

$$
\begin{aligned}
& \left\|\sum_{i \in S_{p}} b_{i} \beta_{i} \bigcup_{j \leqq m}\left(E_{n_{j}}\right)\right\| \\
& \geqq-\sum_{j<p}\left\|\sum_{i \in S_{p}} b_{i} \beta_{i}\left(E_{n_{j}}\right)\right\|+\left\|\sum_{i \in S_{p}} b_{i} \beta_{i}\left(E_{n_{p}}\right)\right\|-\sum_{i \in S_{p}} \sum_{j \geqq n_{p+1}} v\left(\beta_{i}, E_{j}\right) \\
& \quad>-\epsilon\left(1 / 2+1 / 2^{2}+\cdots+1 / 2^{p-1}\right)+3 \epsilon-\epsilon>\epsilon .
\end{aligned}
$$

For $p=m$, the preceding inequalities are easily modified to yield that $\left\|\sum_{i \in S_{p}} b_{i} \beta_{i} \cup_{j \leq m}\left(E_{n_{j}}\right)\right\|>-\epsilon+3 \epsilon=2 \epsilon$. Let $m$ be a positive integer satisfying $m \gamma\left(|\beta|_{8}(S), \epsilon\right)>|\beta|_{8}(S)$, and let $H=\bigcup_{j \leqq m} E_{n_{j}}$. Then

$$
\begin{array}{r}
\|\beta(H)\| \geqq\left\|\sum_{i \leq k_{1}} b_{i} \beta_{i}(H)\right\|+\sum_{j \leq m}\left(\left\|\sum_{i \leq k_{j}} b_{i} \beta_{i}(H)\right\|-\left\|\sum_{i \leqq k_{j-1}} b_{i} \beta_{i}(H)\right\|\right) \\
\geqq m \gamma\left(|\beta|_{s}(S), \epsilon\right)>|\beta|_{s}(S)
\end{array}
$$

which is impossible. 
Let us now turn to a discussion of some Radon-Nikodým theorems. We begin with brief resumé of some pertinent history.

The classical Radon-Nikodým theorem (e.g. [9, Theorem III.10.2]) asserts that if $\Sigma$ is a sigma algebra and $\lambda$ is a countably additive element of $H(C)$ where $C$ denotes the complex numbers, then $\lambda$ can be given an integral representation with respect to a nonnegative, countably additive element $\mu$ of $H(R)$ if, and only if, $\lambda$ is absolutely continuous with respect to $\mu$.

In 1939, S. Bochner published a generalization [1] which removed the restrictions that $\Sigma$ be a sigma algebra and that the set functions be countably additive. A representation for the case where $\mu \in H(R)$ appeared [2] in 1962. Theorem III.10.7 of [9] supplements the classical theorem by allowing $\mu$ to be complex valued, and recently C. Fefferman [10] extended this latter result to the case of a general algebra of subsets of a set. Thereafter, E. Green and the author [7] gave a proof of Fefferman's result, based on [2]. The approach used in [7] will be applied hereinafter to elements of $H(B)$.

While there are several possible definitions of absolute continuity which might be appropriate, we choose the following.

The statement that $\alpha \in H(A), A$ a Banach space, is absolutely continuous with respect to $\beta(\alpha \ll \beta)$ means that if $\epsilon>0$, then there is $\delta>0$ such that if $|\beta|_{s}(E)<\delta$, then $|\alpha|_{s}(E)<\epsilon$.

The statement that $\alpha$ is singular with respect to $\beta(\alpha \perp \beta)$ means that if $\epsilon>0$, then there exists $E \in \Sigma$ such that $|\beta|_{8}(E)<\epsilon$ and $|\alpha|_{s}(S-E)<\epsilon$.

Denote by $L(B, A)$ the space of bounded linear transformations from $B$ to $A$.

The statement that $T$ is a $(\Sigma, M)$-simple function means that $T$ is a function on $S$ to $M$ and, moreover, there is a finite partition $\pi=\left\{E_{i}\right\}_{i \leqq n}$ of $S$ in $\Sigma$ (i.e., $E_{i} \in \Sigma, i \leqq n$ ) such that $T$ is constant on the elements $E_{i}$ of $\pi$.

Henceforth, suppose that $A$ has a basis $\left\{a_{i}\right\}$ of unit vectors.

Theorem 4. Suppose that $A$ is n-dimensional, that $B$ is $m$-dimensional, and that $\alpha \ll \beta$. Then there is a sequence $T_{k}$ of $(\Sigma, L(B, A))$ simple functions such that $\lim _{k}\left|\alpha-\int T_{k} d \beta\right|_{8}(S)=0$.

Proof. From $\alpha \ll \beta$ and $\left|\alpha_{i}(E)\right| \leqq 2 M_{A}|| \alpha(E) \|$ it follows that $\alpha_{i} \ll \beta$. Moreover, letting $\alpha_{i 1}=\alpha_{i} \wedge \beta_{1}$, where $\alpha_{i} \wedge \beta_{1}$ denotes that part of $\alpha_{i}$ which is absolutely continuous with respect to $\beta_{1}$ (see e.g. [2]), and $\alpha_{i j}=\left(\alpha_{i}-\sum_{k<j} \alpha_{i k}\right) \wedge \beta_{j}$ for $i<j \leqq m$, it follows that $\alpha_{i j} \perp \alpha_{i k}$ if $j \neq k$ and also that $\alpha_{i}=\sum_{j \leqq m} \alpha_{i j}$. For $i \leqq n$ and $j \leqq m$ there is a sequence $\left\{f_{i j k}\right\}_{k \geq 1}$ of $(\Sigma, R)$-simple functions such that 


$$
\lim _{k} v\left(\alpha_{i j}-\int f_{i j k} d \beta_{j}, S\right)=0 .
$$

Let $T_{k}(x)$ be the transformation whose matrix with respect to the bases $\left\{a_{i}\right\}$ and $\left\{b_{j}\right\}$ is given by $\left(f_{i j k}(x)\right)_{i, j}$. Then $T_{k}$ is a sequence of $(\Sigma, L(B, A))$ simple functions and $\lim _{k}\left|\alpha-\int T_{k} d \beta\right|_{s}(S)=0$.

In the next theorem it will be convenient to have the following notation. For each positive integer $m$, let $\beta^{m}$ be defined by $\beta^{m}(E)$ $=\sum_{i \unlhd m} b_{i} \beta_{i}(E), E \in \Sigma$. Then $\beta^{m} \in H\left(B^{m}\right)$ where $B^{m}$ denotes the closed linear span, in $B$, of $b_{1}, \cdots, b_{m}$.

Theorem 5. Suppose that $\lim _{m}\left|\beta-\beta^{m}\right|_{s}(S)=0$ and, moreover, that $\alpha \ll \beta$. Then there is an increasing sequence $\left\{m_{k}\right\}$ of positive integers and $a$ sequence $T_{k}$ of $\left(\Sigma, L\left(B^{m_{k}}, A^{k}\right)\right)$-simple functions such that $\left|\alpha^{k}-\int T_{k} d \beta\right|_{8}(S)<1 / k$, where $\int T_{k} d \beta$ is defined to be $\int T_{k} d \beta^{m_{k}}$.

Proof. Let $\epsilon>0$. Then there exists $\delta>0$ such that if $|\beta|_{s}(E)<\delta$, then $|\alpha|_{s}(E)<\epsilon$. Let $m$ satisfy $\left|\beta-\beta^{m}\right|_{s}(S)<\delta / 2$. Then $\left|\beta^{m}\right|_{s}(E)$ $<\delta / 2$ implies that $|\alpha|_{8}(E)<\epsilon$. Notice that $|\beta|_{8}(E)<\delta /\left(2 M_{B}\right)$ implies that $\left|\beta^{m}\right|_{s}(E)<\delta / 2$, and that if $|\alpha|_{s}(E)<\epsilon$, then $\left|\alpha_{i}\right|_{s}(E)<M_{A} \epsilon$. For $k \geqq 1$, choose $\epsilon_{k}$ so that $M_{A} \epsilon_{k}<1 /\left(4 k^{2}\right)$. Then choose $\delta_{k}\left(\delta_{k}<\delta_{k-1}\right)$ and $m_{k}\left(m_{k}>m_{k-1}\right)$ in accordance with the preceding statements. For $i \geqq 1$, let $\alpha_{i 1}=\alpha_{i} \wedge \beta_{1}$ and, for $j>1$, let $\alpha_{i j}=\left(\alpha_{i}-\sum_{p<j} \alpha_{i p}\right) \wedge \beta_{j}$. Let $\gamma_{i j}=\sum_{p \leqq j} \alpha_{i p}$. Then $\alpha_{i j} \perp \alpha_{i p}$ if $j \neq p$, and $\left(\alpha_{i}-\gamma_{i j}\right) \perp \beta_{p}$ for $p \leqq j$. Hence if $\lambda>0$ and $p \leqq j$, then there exists $E_{p} \in \Sigma$ such that $v\left(\beta_{p}, E_{p}\right)<\lambda$ and $v\left(\alpha_{i}-\gamma_{i j}, S-E_{p}\right)<\lambda$. If $F=\bigcap_{p \leq j} E_{p}$, then $v\left(\alpha-\gamma_{i j}, S-F\right)<j \lambda$ and $v\left(\beta_{p}, F\right)<\lambda, p \leqq j$, which implies that $\left|\beta^{j}\right|_{s}(F) \leqq j \lambda$. If $j=m_{k}$ and $\lambda$ satisfies $m_{k} \lambda<\min \left(\delta_{k} / 2,1 /\left(4 k^{2}\right)\right)$, then $v\left(\alpha_{i}-\gamma_{i m_{k}}, S-F\right)<1 /\left(4 k^{2}\right)$ and $\left|\beta^{m_{k}}\right|_{s}(F)<\delta_{k} / 2$. This latter inequality, together with the heretofore assigned properties of $\delta_{k}$, implies that $\left|\alpha_{i}\right|_{s}(F)<1 /\left(4 k^{2}\right)$ and, in turn, that $v\left(\alpha_{i}, F\right)<1 /\left(2 k^{2}\right)$. Then, since $v\left(\alpha_{i}, E\right)=v\left(\alpha_{i}-\gamma_{i j}, E\right)$ $+v\left(\gamma_{i j}, E\right), E \in \Sigma, i, j \geqq 1$, it follows that $v\left(\alpha_{i}-\gamma_{i m_{k}}, F\right)<1 /\left(2 k^{2}\right)$ and, hence, $v\left(\alpha_{i}-\gamma_{i m_{k}}, S\right)<3 /\left(4 k^{2}\right)$ for $i \geqq 1$. Because $\alpha_{i j} \ll \beta_{j}$ there are $(\Sigma, R)$-simple functions $f_{i j k}$ such that $v\left(\alpha_{i j}-\int f_{i j k} d \beta_{j}, S\right)$ $<1 /\left(4 k^{2} m_{k}\right)$. Thus $v\left(\gamma_{i m_{k}}-\int \sum_{j \leqq m_{k}} f_{i j k} d \beta_{j}, S\right)<1 /\left(4 k^{2}\right)$ and, hence, $v\left(\alpha_{i}-\int \sum_{j \leqq m_{k}} f_{i j k} d \beta_{j}, S\right)<1 / k^{2}$. From this latter inequality it follows that if $T_{k}(x)$ is the matrix whose $(i, j)$-th entry is $f_{i j k}(x)$ for $i \leqq k$ and $j \leqq m_{k}$ (and zero otherwise, when necessary, in order that $T_{k}$ have the proper domain and range), then $\left|\alpha^{k}-\int T_{k} d \beta\right|_{s}(S) \leqq 1 / k$.

Corollary 5.1. For each positive integer $n, \lim _{k}\left|\alpha^{n}-\int T_{k} d \beta\right|_{s}(S)=0$.

Corollary 5.2. For each $E \in \sum, \alpha(E)=\lim _{k} \int_{E} T_{k} d \beta$.

Corollary 5.3. If $\lim _{n}\left|\alpha-\alpha^{n}\right|_{s}(S)=0$, then $\lim _{k}\left|\alpha-\int T_{k} d \beta\right|_{s}(S)=0$. 
Example 2 shows that norm convergence of the sequence $\left\{\beta_{i}\right\}$ to zero is not a sufficient condition in order that $\lim \left|\beta-\beta^{n}\right|_{8}(S)=0$. The following example shows that ||$|\beta|||<\infty$ is not a necessary condition in order that $\lim \left|\beta-\beta^{n}\right|_{s}(S)=0$, where $\||\beta|\|=\sup \left\{\Sigma\left\|\beta\left(E_{i}\right)\right\|\right.$; $\pi=\left\{E_{i}\right\}$ is a finite partition of $S$ comprised of elements of $\left.\Sigma\right\}$.

EXAMPLE 3. Suppose that $S$ is the set of positive integers, $\Sigma$ is the algebra of all subsets of $S, B=l_{2}, b_{i}=\left\{\delta_{i j}\right\}_{j \geqq 1}, \beta_{i}(E)=(1 / i) \sum_{j \in E} \delta_{i j}$, and $\beta(E)=\sum b_{i} \beta_{i}(E)$. Then $\beta \in H(B),\left\{b_{i}\right\}$ satisfies condition $G_{2}$, $\||| \beta \mid\|=\infty$ and $\lim \left|\beta-\beta^{n}\right|_{8}(S)=0$.

We conclude with conditions which are sufficient to insure that $\lim \left|\beta-\beta^{n}\right|_{s}(S)=0$.

Theorem 6. Suppose that $\||\beta|\|=M<\infty$ and that $\left\{b_{i}\right\}$ satisfies condition $G_{2}$. Moreover suppose that if

$$
x_{k}=\sum_{i \leqq n} b_{i} \lambda_{k i} \text { and } y_{k}=\sum_{n<i \leqq m_{k}} b_{i} \lambda_{k i}, \quad k \leqq m,
$$

then

$$
\left\|\sum_{k \leqq m}\left(x_{k}+y_{k}\right)\right\|-\left\|\sum_{k \leqq m} x_{k}\right\| \leqq \sum_{k \leqq m}\left(\left\|x_{k}+y_{k}\right\|-\left\|x_{k}\right\|\right) .
$$

Then $\lim \left|\beta-\beta^{n}\right|_{s}(S)=0$.

Proof. Let $\epsilon>0$. Then let $\delta$ be a positive number such that if $\left\|\sum_{i \leqq n} b_{i} \lambda_{i}\right\| \leqq M$ and $\left\|\sum_{n<i \leq m} b_{i} \lambda_{i}\right\| \geqq \epsilon$, then $\left\|\sum_{i \leq m} b_{i} \lambda_{i}\right\|$ $\geqq\left\|\sum_{i \leqq n} b_{i} \lambda_{i}\right\|+\delta$. Let $\pi=\left\{E_{i}\right\}$ be a finite partition of $S$ in $\Sigma$ satisfying $\sum_{i}\left\|\beta\left(E_{i}\right)\right\|>M-\delta / 2$. Let $n$ be a positive integer satisfying $\sum_{i}\left\|\left(\beta-\beta^{k}\right)\left(E_{i}\right)\right\|<\delta / 2$ if $k \geqq n$, and let $k \geqq n$. Then $\sum_{i}\left\|\beta^{k}\left(E_{i}\right)\right\|$ $>M-\delta$. Suppose that $\left|\beta-\beta^{k}\right|_{s}(S)>\epsilon$. Then there is an element $E$ of $\Sigma$ satisfying $\left\|\left(\beta-\beta^{k}\right)(E)\right\|>\epsilon$ and, hence, $\left(\|\beta(E)\|-\left\|\beta^{k}(E)\right\|\right) \geqq \delta$. Let $\pi_{1}$ be the partition of $S$ generated by $\pi$ and $E$, let $F_{i}=E_{i} \cap E$ and let $G_{i}=E_{i}-E$. Then the contradiction

$$
\begin{aligned}
\sum_{T \in \pi_{1}}\|\beta(T)\|= & \sum_{T \in \pi_{1}}\left\|\beta^{k}(T)\right\|+\sum_{i}\left(\left\|\beta\left(F_{i}\right)\right\|-\left\|\beta^{k}\left(F_{i}\right)\right\|\right) \\
& +\sum_{i}\left(\left\|\beta\left(G_{i}\right)\right\|-\left\|\beta^{k}\left(G_{i}\right)\right\|\right) \geqq \sum_{E \in \pi}\left\|\beta^{k}(E)\right\| \\
& +\left(\|\beta(E)\|-\left\|\beta^{k}(E)\right\|\right)>(M-\delta)+\delta=M
\end{aligned}
$$

obtains.

\section{REFERENCES}

1. S. Bochner, Additive set functions on groups, Ann. of Math. 40 (1939), 769-799.

2. R. B. Darst, $A$ decomposition of finitely additive set functions, J. Math. Reine Angew. 210 (1962), 31-37. 
3. - A decomposition for complete normed abelian groups with applications to spaces of additive set functions, Trans. Amer. Math. Soc. 103 (1962), 549-559.

4. —, The Lebesgue decomposition, Duke Math. J. 30 (1963), 553-556.

5. - $A$ direct proof of Porcelli's condition for weak convergence, Proc. Amer. Math. Soc. 16 (1965), 1094-1096.

6. - On a theorem of Nikodym with applications to weak convergence and von Neumann algebras, Pacific J. Math. 23 (1967), 473-477.

7. R. B. Darst and E. Green, On a Radon-Nikodym theorem for finitely additive set functions, Pacific J. Math. 27 (1968), 255-259.

8. M. M. Day, Normed linear spaces, 2nd rev. ed., Ergebenesse der Math., Heft 21, Springer-Verlag, Berlin, 1962.

9. N. Dunford and J. T. Schwartz, Linear operators, Interscience, New York, 1958.

10. C. Fefferman, $A$ Radon-Nikodým theorem for finitely additive set functions, Pacific J. Math. 23 (1967), 35-45.

11. P. Porcelli, Two embedding theorems with applications to weak convergence and compactness in spaces of additive type functions, J. Math. Mech. 9 (1960), 273-292.

Purdue University 